\title{
Uso de la técnica de micromatrices de tejido en histología veterinaria
}

\author{
Use of tissue microarrays in veterinary histology
}

Sanz Ressel $\mathrm{BL}^{1,2^{*}}$, Massone $\mathrm{AR}^{3}$, Barbeito $\mathrm{CG}^{1,2}$

'Laboratorio de Histología y Embriología Descriptiva, Experimental y Comparada, Facultad de Ciencias Veterinarias (FCV), Universidad Nacional de La Plata(UNLP); ${ }^{2}$ FCV, CONICET, UNLP, La Plata, Argentina; ${ }^{3}$ Laboratorio de Patología Especial Veterinaria Dr. B. Epstein, FCV, UNLP. ${ }^{*}$ Correo electrónico del autor: Isanzressel@fcv.unlp.edu.ar

\begin{abstract}
Resumen: Las micromatrices de tejido son plataformas de alto rendimiento que permiten el análisis de decenas a cientos de muestras de tejidos en forma simultánea. Estas se han utilizado, en especial, para analizar tejidos neoplásicos. Sin embargo, los beneficios de su aplicación pueden ser aprovechados en otras áreas de investigación, como la embriología y la histología. El presente trabajo explora el uso de la técnica de micromatrices de tejido para el análisis morfológico y molecular de tejidos animales normales. Un total de 82 muestras de piel canina normal prenatal y posnatal fueron utilizadas para construir la matriz. A partir de ésta, se obtuvo una gran cantidad de secciones en las que se aplicaron técnicas de histología convencional y de inmunohistoquímica. Los resultados nos muestran la eficacia de la técnica para realizar el análisis morfológico y molecular de decenas de muestras de tejido normal en forma simultánea. Esto permite la evaluación más estandarizada de los tejidos, reduciendo así la variabilidad que puede ocurrir cuando se realizan ensayos sobre muestras individuales.
\end{abstract}

Palabras clave: micromatrices de tejido, inmunohistoquímica, piel, caninos

\begin{abstract}
Tissue microarrays are platforms for high throughput simultaneous analysis of tens to hundreds of tissue samples. They have been used especially to analyze neoplastic tissues. However, the advantages of this technique could be applied to other research areas such as embryology and histology. This study explores the use of tissue microarrays for morphological and molecular analysis of normal tissues from animals. A total of 82 samples of normal skin from prenatal and postnatal dogs were selected and used to construct the array. A large number of sections from this array block were obtained and routine stain and immunohistochemistry techniques were applied. The results show the effectiveness of the technique for morphological and molecular analysis of a large number of normal tissue samples simultaneously. This allows the standardized assessment of tissues, thus reducing the variability that can occur when testing is performed on individual samples.
\end{abstract}

Key words: tissue microarrays, immunohistochemistry, skin, dogs 


\section{Introducción}

Las micromatrices de tejidos incluidos en parafina (MMsT) son plataformas de alto rendimiento que permiten el análisis de múltiples muestras de tejidos en forma simultánea (Hewitt 2004). Estas plataformas se construyen mediante la obtención de biopsias cilíndricas a partir de decenas a cientos de bloques de parafina donantes, las cuales son reincorporadas en un único bloque de parafina receptor (Kononen et al. 1998). Por lo tanto, una sección obtenida a partir de esta matriz contiene pequeñas muestras de tejido representativas de los diferentes casos, sobre la cual se pueden aplicar las mismas técnicas que se usarían sobre una sección de tejido convencional en un portaobjetos, como por ejemplo la inmunohistoquímica (IHQ) (Hewitt 2004; Kononen et al. 1998).

Una micromatriz de tejidos (MMT) bien diseñada permite ahorrar tiempo y materiales, así como también facilita el análisis simultáneo de dianas moleculares en condiciones idénticas y estandarizadas sobre muestras múltiples, reduciendo la variabilidad de los experimentos al evitar la necesidad de realizarlos una y otra vez (Hewitt 2004). Por ejemplo, una sola inmunotinción puede proporcionar información valiosa sobre todas las muestras que se encuentran sobre el portaobjetos en forma simultánea, mientras que las secciones posteriores de la matriz pueden ser utilizadas para analizar otros anticuerpos (Molinolo et al. 2007). Estas ventajas llevaron a que la aplicación de esta técnica surja como una herramienta esencial en el campo de la oncología, en la que ha permitido la detección rápida y rentable de biomarcadores de utilidad diagnóstica, pronóstica y predictiva (Hewitt 2009; Hewitt 2012; Kononen et al. 1998).

Aunque la técnica de MMsT fue descripta inicialmente para la investigación del cáncer y se utiliza principalmente en este campo, la posibilidad de su aplicación se ha ampliado hacia otras áreas de investigación (Avninder et al. 2008). Así, sus beneficios pueden ser aprovechados en estudios en los que se trabaje, por ejemplo, con múltiples muestras de tejido normal (Kononen et al. 1998), como la embriología y la histología. En estas áreas, la técnica de MMsT surge como una alternativa atractiva para la investigación de aspectos morfológicos y funcionales, dado que, para realizar un aporte de relevancia es indispensable contar con una gran cantidad de muestras en las que se realicen los análisis en forma estandarizada.

El presente trabajo explora por primera vez el uso de la técnica de MMsT en el estudio de la piel canina durante el desarrollo ontogénico, a partir de una gran cantidad de muestras provenientes de diferentes regiones corporales de fetos y adultos.

\section{Materiales y métodos \\ Micromatriz de tejido}

Se tomaron muestras de piel a partir de distintas regiones corporales (dorso, flanco, vientre, dedo, plano nasal, pabellón auricular y, en los machos, escroto) de fetos y adultos caninos. Las muestras de fetos provinieron de histerectomías realizadas por médicos veterinarios de actividad privada y, las de animales adultos, de caninos ingresados al Servicio de Anatomía Patológica de la Facultad de Ciencias Veterinarias (Universidad Nacional de La Plata). Las muestras fueron procesadas para su inclusión en parafina y su posterior coloración con la técnica de hematoxilina y eosina (H-E). Luego de la observación de las secciones coloreadas con H-E, un total de 82 bloques de parafina conteniendo los tejidos de interés (bloques donantes) fueron seleccionados para la posterior construcción de la MMT. Cuatro bloques de parafina adicionales, conteniendo muestras correspondientes a otros órganos, fueron seleccionados para ser incluidos como controles en la matriz.

El método utilizado en la construcción de la MMT fue el descripto por Hewitt (2004), utilizando un arrayer de tejido semiautomático (TM Arrayer TM, Pathology Devices, Inc.) y un diámetro de cilindro (núcleo) de $1 \mathrm{~mm}$. Sobre cada una de las secciones coloreadas con $\mathrm{H}-\mathrm{E}$, correspondiente a cada bloque donante, se procedió a la marcación de las áreas de tejido de interés utilizando un marcador indeleble bajo observación microscópica. Luego, se ordenó cada bloque donante con su sección correspondiente para la posterior construcción de la matriz. Un bloque de parafina receptor fue construido utilizando un molde específico y parafina bajo punto de fusión. El bloque de parafina receptor se colocó entonces en el arrayer de tejido y se procedió a la construcción de la MMT. Sobre un soporte específico se ubicó el bloque donante y sobre éste la sección coloreada con H-E correspondiente. El conjunto formado por soporte-bloque donante-sección coloreada con $\mathrm{H}-\mathrm{E}$ se alineó de forma tal que el área de interés marcada sobre el portaobjetos se ubicara debajo de la aguja de perforación. Se retiró el portaobjetos y se procedió a la obtención de una biopsia cilíndrica (núcleo) a partir del bloque de parafina donante. Se retiró el soporte que contenía el bloque donante y se procedió a reinsertar el núcleo contenido en la aguja de perforación en el bloque de parafina receptor. Este procedimiento se repitió con cada uno de los bloques donantes. Una vez finalizada la inserción de los núcleos de interés, se colocó la MMT en una estufa a $37{ }^{\circ} \mathrm{C}$ durante $10 \mathrm{~min}$ y se presionó su superficie. Este último procedimiento se repitió tres veces. Finalmente, se realizó el templado de la MMT en una estufa a $37^{\circ} \mathrm{C}$. Una vez concluida la MMT, se obtuvieron secciones que se colorearon 
con $\mathrm{H}-\mathrm{E}$ para realizar un análisis general de la matriz y evaluar su calidad.

\section{Inmunohistoquímica}

Se utilizaron dos secciones de la MMT para la aplicación de la técnica de IHQ utilizando un anticuerpo específico anti $\beta$-catenina ( $\beta$-Catenin D10A8XP тм Rabbitm Ab \#8480; CellSignaling Technologies; dilución 1:100).

Los portaobjetos que contenían las secciones de la MMT se colocaron en una estufa a $65^{\circ} \mathrm{C}$ durante 30 min para fundir la parafina, se sumergieron en Safeclear II (Fisher Scientific) para el desparafinado durante $5 \mathrm{~min}$ (tres veces) y se hidrataron mediante pasajes sucesivos en alcoholes de graduación decreciente. Para bloquear la peroxidasa endógena se incubaron en peróxido de hidrógeno al $3 \%$ en alcohol $70^{\circ}$ durante 30 min y se lavaron con agua destilada durante $5 \mathrm{~min}$, tres veces. Para la recuperación antigénica, los portaobjetos se colocaron en un recipiente que contenía ácido cítrico $10 \mathrm{mM}$ en un horno de microondas durante 20 min ( 2 min a $100 \%$ de potencia y 18 min a $20 \%$ de potencia). Los portaobjetos se dejaron enfriar durante $15 \mathrm{~min}$ a temperatura ambiente, se lavaron una vez con agua destilada y luego tres veces con PBS. Las secciones se incubaron en solución de bloqueo (albúmina sérica bovina al 2,5\% en PBS) durante $30 \mathrm{~min}$ a temperatura ambiente. El exceso de solución se descartó y las secciones se incubaron con el anticuerpo primario diluido en solución de bloqueo a $4{ }^{\circ} \mathrm{C}$ durante la noche. Después de lavar con PBS, los portaobjetos se incubaron secuencialmente con el anticuerpo secundario biotinilado (Biotinylated Goat Anti-Rabbit IgG Antibody; BA-1000; Vector Laboratories; 1: 400) durante 30 min y luego con el complejo avidina-biotina (VECTASTAIN® Elite $®$ ABC Kit; PK-6100; Vector Laboratories) durante 30 min a temperatura ambiente. Los portaobjetos se lavaron y se revelaron con 3,3'-diaminobencidina (SIGMAFAST ${ }^{\mathrm{TM}}$ 3,3'-Diaminobenzidine tablets; D4168-50SET; Sigma Chemical) bajo control microscópico. La reacción se detuvo con agua destilada y los tejidos se contrastaron con hematoxilina de Mayer, para luego continuar con el deshidratado y montado.El anticuerpo utilizado fue probado previamente en muestras de piel canina normal para confirmar su reactividad en la especie, así como también, para establecer las condiciones óptimas para su uso.

\section{Resultados}

\section{Diseño y construcción de la matriz}

Un total de 110 muestras de piel canina normal fueron obtenidas y procesadas. Luego de la observación de las secciones coloreadas con $\mathrm{H}-\mathrm{E}$, se selec- cionaron 82 muestras para la posterior construcción de la MMT. La causa más común de exclusión fue la mala fijación del tejido.

Dado que las muestras eran en general pequeñas y morfológicamente homogéneas, fue sencillo marcar en las secciones coloreadas con H-E las zonas precisas de los bloques donantes desde donde la aguja de perforación podría obtener los tejidos. El tiempo empleado para llevar a cabo esta selección fue de aproximadamente 3 horas, mientras que el necesario para llevar a cabo el diseño y construcción de la MMT fue de aproximadamente 6 horas. Los casos fueron distribuidos en la matriz teniendo en cuenta su edad de desarrollo, dejando los controles en el margen inferior derecho. En la figura $1(A)$ se muestra la MMT finalizada.

En las secciones obtenidas a partir de la MMT sobre las que se aplicó la técnica de $\mathrm{H}$-E para realizar un análisis general de la matriz y evaluar su calidad, se confirmó la presencia de los tejidos de interés (epidermis, dermis y tejido subcutáneo) en todos los núcleos. Las sucesivas secciones de la matriz obtenidas a diferentes profundidades presentaron características tisulares similares entre sí, comparables a las que se obtendrían trabajando con los bloques de parafina convencionales. Asimismo, después de la aplicación de la técnica de H-E se observó una mínima pérdida de los núcleos con los tejidos de interés, siendo esta de 2 o 3 núcleos en cada sección. Los resultados de la aplicación de la técnica de H-E sobre una sección de la MMT se muestran en la figura 1 ( $B$ y $C$ ). Por último, se realizó la comparación de las secciones coloreadas con H-E obtenidas a partir de la matriz con las respectivas secciones completas provenientes de los bloques de parafina donantes, observándose una concordancia de tejido entre los núcleos de la matriz con las respectivas secciones completas en todos los casos (Figura 2).

\section{Inmunohistoquímica}

En las secciones obtenidas a partir de la MMT sobre las que se realizó IHQ para evaluar la expresión de $\beta$-catenina se observó una intensa marcación en las células de la epidermis, así como también en el folículo piloso y en las células de las glándulas sebáceas y sudoríparas apocrinas. Esta expresión tuvo una distribución citoplasmática y de membrana. Por otra parte, se realizó la comparación de la expresión de $\beta$-catenina obtenida en las secciones de la matriz con algunas secciones completas provenientes de los bloques de parafina donantes, observándose una concordancia en la marcación entre los núcleos de la matriz con las respectivas secciones completas en los casos analizados. Asimismo, la calidad de los diferentes núcleos de tejido fue en general buena 

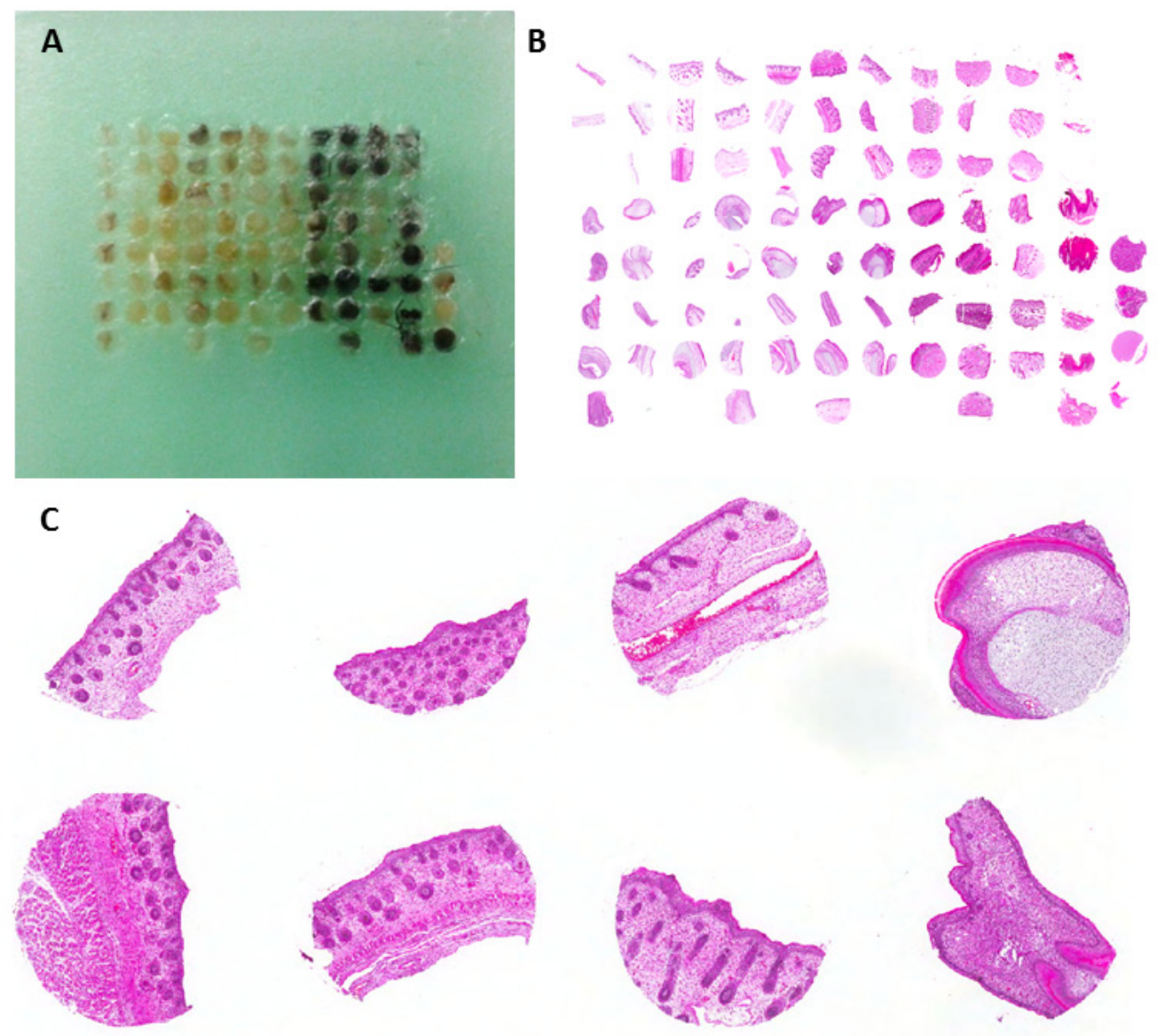

Figura 1. Micromatriz de tejidos construida a partir de muestras de piel canina normal. (A) Imagen de la micromatriz de tejido finalizada, conteniendo los núcleos de $1 \mathrm{~mm}$ de diámetro obtenidos a partir de muestras de piel canina normal prenatal y posnatal. (B) Sección de la MMT coloreada con H-E. (C) Imágenes correspondientes a algunos núcleos representativos de la matriz para mostrar la calidad de los mismos. Objetivo 2x.

tras la aplicación de IHQ, observándose una mínima pérdida de los mismos (2-3 núcleos). Ejemplos de los resultados de la aplicación de esta técnicase muestran en la figura 3.

\section{Discusión}

El uso de la técnica de MMsT brinda una serie de beneficios cuando se desea trabajar con grandes colecciones de tejido. La posibilidad de incluir en un único bloque de parafina decenas a cientos de muestras permite no solo ahorrar tiempo y materiales, sino también reducir la variabilidad de los experimentos que se realicen posteriormente (Hewitt 2004; Molinolo et al. 2007). El principal beneficio observado fue la reducción notable en el tiempo y la cantidad de materiales necesarios para el desarrollo de la técnica de IHQ. Esto se debe a que en una sola inmunotinción se pudo obtener información de las 82 muestras de piel que se encontraban sobre el portaobjetos en forma simultánea. Si esta técnica se hubiese aplicado sobre las secciones completas, no sólo se hubiesen incrementado los costos y materiales necesarios para su desarrollo, dado que 82 secciones hubiesen sido necesarias, sino que además no hubiese sido posible la aplicación de la técnica de IHQ en forma simultánea para esta cantidad de muestras.

La técnica de MMsT presenta algunas limitaciones cuando se utilizan tejidos neoplásicos para su construcción, dado que cada núcleo representa una pequeña fracción de la lesión (Kononen et al. 1998). Así, en las matrices construidas a partir de este tipo de tejidos, las secciones tomadas a diferentes profundidades muestran características ligeramente diferentes o, como sucede en algunos casos, la ausencia total de tejido tumoral en algunos núcleos cuando se alcanzan los límites no neoplásicos (Molinolo et al. 2007). En este estudio, observamos que las MMsT construidas a partir de tejidos normales no presentan esta limitación. Las sucesivas secciones coloreadas con H-E obtenidas a diferentes profundidades de la matriz presentaron características tisulares similares entre 

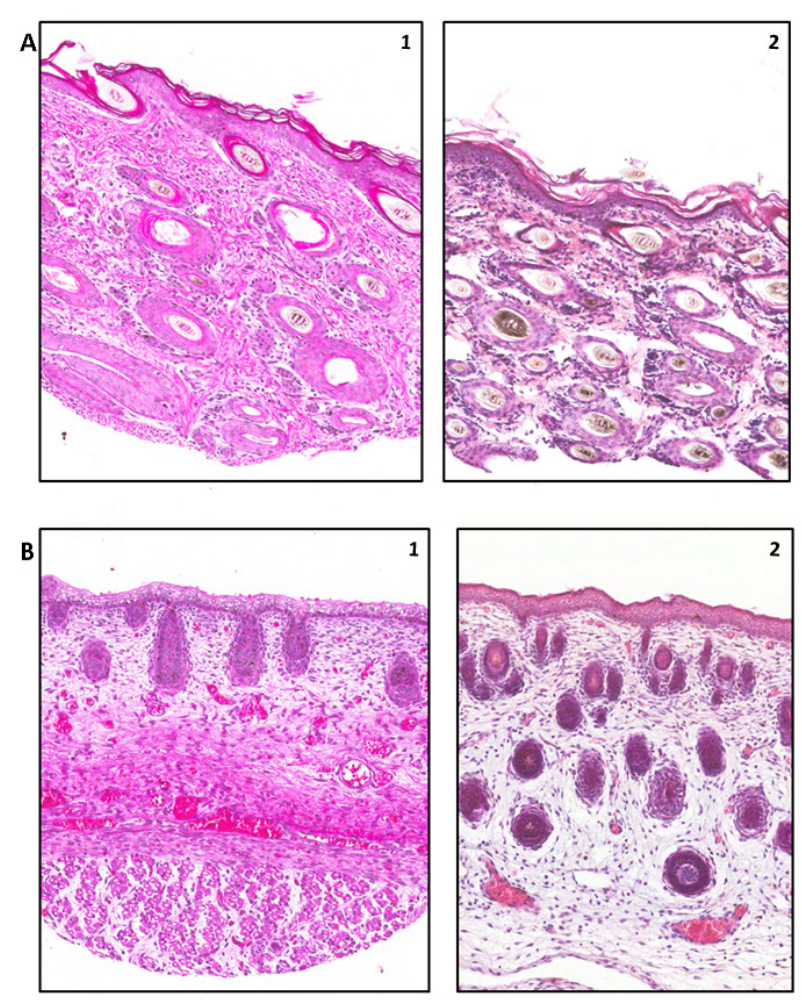

Figura 2.Imágenes representativas de secciones de la MMT coloreadas con H-E y su correlación con las imágenes correspondientes a las secciones completas. (A) Imágenes correspondientes a piel de la región del dorso de un canino neonato: (1) núcleo de la MMT y (2) sección convencional. Objetivo 10x. (B) Imágenes correspondientes a piel de la región del dorso de un canino de 50 días de gestación: (1) núcleo de la MMT y (2) sección convencional. Objetivo 10X.

sí, comparables a las que se obtendrían trabajando con los bloques de parafina convencional.

Para evaluar la viabilidad del uso de la técnica de MMsT para estudiar las complejas redes de señalización involucradas en el desarrollo de la piel canina normal, se procedió a la utilización de técnicas de IHQ sobre secciones obtenidas a partir de la MMT. El anticuerpo seleccionado fue anti $\beta$-catenina debido a que ha sido establecida previamente como una molécula relevante en el desarrollo de la piel normal en los caninos (Bongiovanni et al. 2011). En las secciones obtenidas a partir de la MMT se observó una intensa expresión citoplasmática y de membrana de $\beta$-catenina en los distintos núcleos de tejido. Este patrón de marcación fue similar al encontrado utilizando la técnica de inmunohistoquímica sobre secciones completas, lo cual indica que la técnica de MMsT resulta de utilidad para detectar el nivel de expresión de esta molécula sobre muestras múltiples.

Aunque la técnica de MMsT ha sido ampliamente utilizada para la evaluación inmunohistoquímica de
A
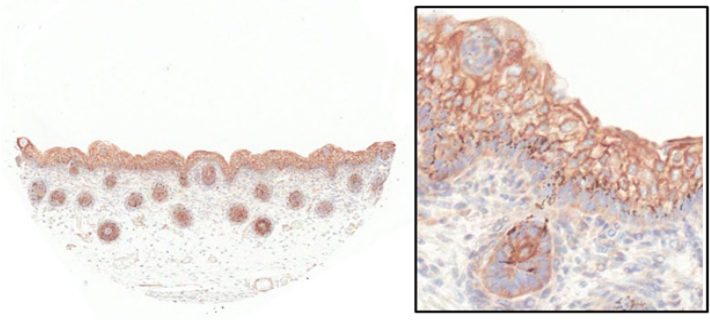

B
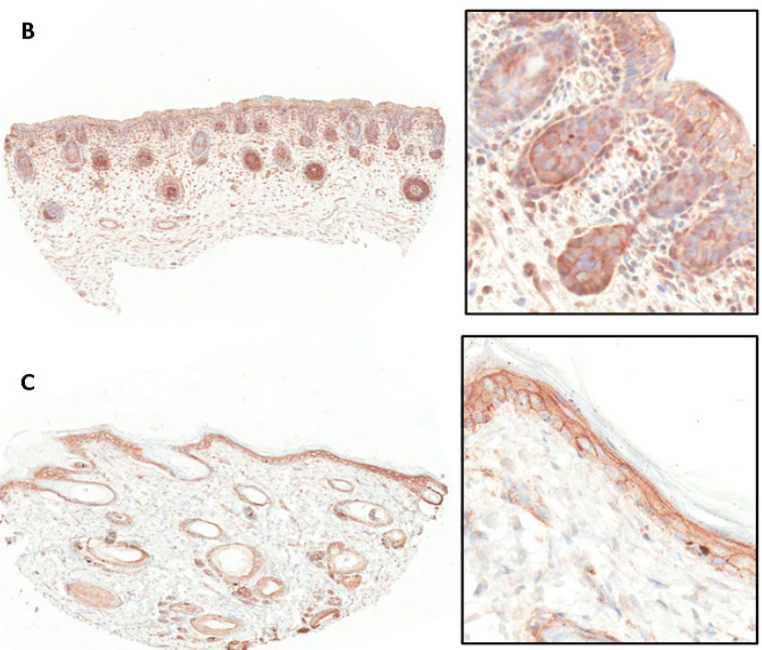

Figura 3. Imágenes representativas de la aplicación de IHQ para la marcación de $\beta$-catenina sobre secciones de la MMT. (A) Imágenes correspondientes a piel de la región del dorso de un canino de 45 días de gestación. (B) Imágenes correspondientes a piel de la región del dorso de un canino de 50 días de gestación. (C) Imágenes correspondientes a piel de la región del dorso de un canino neonato. En los recuadros se muestran imágenes de $(A),(B)$ y $(C)$, respectivamente, con objetivo 40X.

muestras de tejidos en medicina humana, y a pesar de que los tejidos animales son ampliamente utilizados para su construcción en investigación (Braunschweig et al. 2005), existen relativamente pocos informes sobre su uso en medicina veterinaria, estando estos restringidos al campo de la oncología (Buishand et al. 2014; Hammer et al. 2007; Higgins et al. 2010; Keller et al. 2007; Khanna et al. 2002; Muscatello et al. 2015; Wohlsein et al. 2012). El presente estudio es, según nuestro conocimiento, el primero en validar el uso de MMsT en investigación en áreas básicas de medicina veterinaria, como son la embriología y la histología. Así, la técnica de MMsT surge como una alternativa viable cuando se desea realizar un estudio ontogénico de un órgano como la piel canina, dado que es imprescindible disponer de decenas a cientos de muestras en las que aspectos morfológicos y funcionales puedan ser evaluados en forma simultánea.

Este trabajo podrá profundizarse mediante un análisis morfológico exhaustivo y la aplicación de técnicas de IHQ que permitan detectar moléculas implica- 
das en las distintas vías de señalización involucradas en el desarrollo de la piel canina normal. Esto no solo resultará de utilidad para entender la naturaleza de los mecanismos del desarrollo normal, sino que también podrán ser relevantes en el área de patología, para comprender los mecanismos desregulados que subyacen al desarrollo de enfermedades originadas en este órgano en el adulto.

\section{Agradecimientos}

Agradecemos especialmente al Dr. Alfredo Molinolo, al Dr. Stephen Hewitt y a su equipo de trabajo por su colaboración en el presente estudio.

\section{Conflicto de intereses}

Todos los autores declaran que no existe conflicto de intereses, relaciones financieras, personales o de otro tipo con personas u organizaciones que pudieran afectar al presente trabajo.

\section{Bibliografía}

Avninder S, Ylaya K, Hewitt SM. 2008. Tissue microarray: a simple technology that has revolutionized research in pathology. Journal of postgraduate medicine. 54(2):158-62.

Bongiovanni L, Malatesta D, Brachelente C, D’Egidio S, Della Salda L. 2011. $\beta$-catenin in canine skin: immunohistochemical pattern of expression in normal skin and cutaneous epithelial tumours. Journal of comparative pathology. 145(2-3):138-47.

Braunschweig T, Chung JY, Hewitt SM. 2005. Tissue microarrays: bridging the gap between research and the clinic. Expert review of proteomics. 2(3):325-36.

Buishand FO, Visser J, Kik M, Gröne A, Keesler RI, Briaire-de Bruijn IH, Kirpensteijn. 2014. Evaluation of prognostic indicators using validated canine insulinoma tissue microarrays. Veterinary Journal. 201(1):57-63.

Hammer AS, Williams B, Dietz HH, Hamilton-Dutoit SJ. 2007. High-throughput immunophenotyping of 43 ferret lymphomas using tissue microarray technology. Veterinary Pathology. 44(2):196-203.

Hewitt SM. 2004. Design, construction, and use of tissue microarrays. Methods Molecular Biology. 264:61-72.

Hewitt SM. 2009. Tissue microarrays as a tool in the discovery and validation of tumor markers. Methods Molecular Biology. 520(8):151-61.

Hewitt SM. 2012. Tissue microarrays as a tool in the discovery and validation of predictive biomarkers. Methods Molecular Biology. 823:201-14.

Higgins RJ, Dickinson PJ, LeCouteur RA, Bollen AW, Wang H, Wang H, Corely LJ, Moore LM, Zang W, Fuller GN. 2010. Spontaneous canine gliomas: overexpression of EGFR, PDGFRa and IGFBP2 demonstrated by tissue microarray immunophenotyping. Journal of Neurooncology. 98(1):49-55.

Khanna C, Prehn J, Hayden D, Cassaday RD, Caylor J, Jacob S, Bose SM, Hong SH, Hewitt SM, Helman LJ. 2002. A randomized controlled trial of octreotide pamoate long- acting release and carboplatin versus carboplatin alone in dogs with naturally occurring osteosarcoma: evaluation of insulin-like growth factor suppression and chemotherapy. Clinical Cancer Research. 8(7); 2406-12.

Keller SM, Keller BC, Grest P, Börger CT,Guscetti F. 2007. Validation of tissue microarrays for immunohistochemical analyses of canine lymphomas. Journal of veterinary diagnostic investigation. 19(6):652-9.

Kononen J, Bubendorf L, KallioniemiA,Bärlund M, Schraml P, Leighton S, Torhorst J, Mihatsch MJ,Sauter G, Kallioniemi OP. 1998. Tissue microarrays for high-throughput molecular profiling of tumor specimens. Nature Medicine. 4(7):844-7.

Molinolo AA, Hewitt SM, Amornphimoltham P, Keelawat S, Rangdaeng S, Meneses Garcia A, Raimondi AR, Jufe R, Itoiz M, Gao Y, Saranath D, Kaleebi GS, Yoo GH, Leak L, Myers EM, Shintani S, Wong D, Massey DH, Yeudall WA, Lonardo F, Ensley J, Gutkind JS. 2007. Dissecting the Akt/ mammalian target of rapamycin signaling network: emerging results from the head and neck cancer tissue array initiative. Clinical Cancer Research. 13(17):4964-73.

Muscatello LV, Sarli G, Beha G, Asproni P, Millanta F, Poli A, De Tolla LJ, Benazzi C, Brunetti B. 2015. Validation of tissue microarray for molecular profiling of canine and feline mammary tumours. Journal of Comparative Patholology. 152(2-3):153-60.

Wohlsein P, Recker T, Rohn K, Baumgärtner W. 2012. Validation of usefulness of tissue microarray technology in primary tumours of the canine and feline central nervous system. Journal of Comparative Patholology. 146(4):320-6. 